\title{
A COMPLEX SPACE WHOSE SPECTRUM IS NOT LOCALLY COMPACT ANYWHERE
}

\author{
SANDRA HAYES AND JEAN-PIERRE VIGUÉ
}

\begin{abstract}
An example of a two-dimensional complex space is given with the property that the continuous spectrum of the global holomorphic functions is not locally compact at any point.
\end{abstract}

Introduction. The spectrum $S_{c}(O(X))$ of the global holomorphic functions $O(X)$ on a complex space $(X, O)$ is the set of all continuous complex-valued algebra homomorphisms on $O(X)$ endowed with the Gelfand topology. This functional analytic concept has important applications to fundamental problems in complex analysis. For instance, due to a theorem of Igusa-Remmert-Iwahashi-Forster (see $[3,1.5])$, if there is at least one point where the spectrum $S_{c}(O(X))$ is not locally compact, then the complex space $(X, O)$ does not have a Stein envelope of holomorphy. ${ }^{1}$

In all the examples known of complex spaces $(X, 0)$ without a Stein envelope of holomorphy, the set of points in the spectrum $S_{c}(O(X))$ where local compactness fails is a nonempty, extremely small, proper subset of $S_{c}(O(X))$; frequently, it consists of just one point $[2,5.3 ; 7,4.2,4.3]$. A natural question is whether such pathological points can be described as a thin subset of the spectrum. However, the purpose of this note is to show that the spectrum $S_{c}(O(X))$ of a complex space $(X, O)$ need not be locally compact at any point at all; in the example constructed here, $X$ is two dimensional. A complex space always refers to a reduced complex space with countable topology.

Construction. The example will be constructed in two steps. The main idea of the first step is to find a two-dimensional complex space $(X, O)$ whose spectrum $S_{c}(O(X))$ has the following description. Take a two-dimensional complex plane and attach infinitely many two-dimensional complex planes transversally along every line of a countable dense subset of lines in the first plane. In the second step, the unique position of the initial plane will be eliminated by carrying out the construction of the first step for every plane attached to the initial plane.

First step of the construction. For every nonnegative integer $n \in \mathbf{N}$, set

$$
S_{n}:=\left\{(x, y) \in \mathbf{C}^{2}:|x| \leq n+1, n \leq|y| \leq n+\frac{1}{2}\right\} .
$$

Let $D$ be the Reinhardt domain in $\mathbf{C}^{2}$ obtained by removing all the sets $S_{n}, n \in \mathbf{N}$, from $\mathbf{C}^{2}$.

Received by the editors August 19, 1985.

1980 Mathematics Subject Classification (1985 Revision). Primary 32D10; Secondary $46 \mathrm{HO5}$.

${ }^{1}$ Whether the converse of this statement holds is open. 
Take countably many copies $D_{n}, n \in \mathbf{N}$, of $D$. Fix $D_{0}$, and for $n \geq 1$ consider each $D_{n}$ as being transversal to $D_{0}$ in the following way. Choose a dense sequence $\left(\alpha_{n}\right)_{n \in \mathbf{N}}$ in $\mathbf{C}$ with $\left|\alpha_{n}\right|<n+1$ for $n \in \mathbf{N}$. For $\mathbf{N}^{*}:=\mathbf{N} \backslash\{0\}$ let $\varphi: \mathbf{N}^{*} \rightarrow \mathbf{N}$ be a surjective map where each value is assumed countably many times and $\varphi(n)<n$ holds for every $n \in \mathbf{N}^{*}$. The existence of such a map can be seen by taking for every $n \in \mathbf{N}^{*}$ a $p \in \mathbf{N}$ with $p^{2} \leq n<(p+1)^{2}$ and then defining $\varphi(n):=n-p^{2}$. In $\mathbf{C}^{3}$ with coordinates $x, y, z$, set $D_{0}:=D \times\{0\}$. Let $D_{m}$ be the Reinhardt domain in $\left\{\alpha_{\varphi(m)}\right\} \times \mathbf{C}^{2}$ which remains after removing for every $n \in \mathbf{N}$ the set $\left\{\alpha_{\varphi(m)}\right\} \times S_{n}^{\prime}$, where

$$
S_{n}^{\prime}:=\left\{(y, z) \in \mathbf{C}^{2}: n \leq|y| \leq n+\frac{1}{2},|z| \leq n+1\right\} .
$$

Roughly speaking, the desired complex space $X$ is defined by attaching each $D_{n}$ for $n \geq 1$ to $D_{0}$ along

$$
R_{n}:=\left\{(x, y, 0) \in \mathbf{C}^{3}: x=\alpha_{\varphi(n)}, n+\frac{1}{2}<|y|<n+1\right\} .
$$

More precisely, observe that, for $n \in \mathrm{N}^{*}, R_{n}$ is always an analytic subset of $D_{n}$; since $\left|\alpha_{\varphi(n)}\right|<n+1$ is valid, $R_{n}$ is also an analytic subset of $D_{0}$. For every $n \in \mathbf{N}^{*}$, a complex space $X_{n}$ having $R_{n+1}$ as an analytic subset will be defined by induction.

Let $X_{1}$ be the complex space obtained by attaching $D_{1}$ to $D_{0}$ along $R_{1}$. This space

$$
X_{1}:=D_{0}+_{R_{1}} D_{1}
$$

is the fiber sum (pushout) of $D_{0}$ and $D_{1}$ under the inclusion $R_{1} \rightarrow D_{0}$ and $R_{1} \rightarrow D_{1}$ [6]. Define

$$
X_{n}:=X_{n-1}+R_{n} D_{n}
$$

as the fiber sum of $X_{n-1}$ and $D_{n}$ under the inclusions $R_{n} \rightarrow X_{n-1}$ and $R_{n} \rightarrow D_{n}$.

For $n \in \mathbf{N}^{*}$ let $\iota_{n}: X_{n} \rightarrow X_{n+1}$ be the inclusion; $\iota_{n}$ embeds $X_{n}$ in $X_{n+1}$ as a closed subspace. Denote by $X$ the direct limit of the expanding system $\left(X_{n}, \iota_{n}\right)$, i.e.

$$
X:=\underset{\lim }{\longrightarrow} X_{n}
$$

With the direct limit topology, $X$ is a Hausdorff space $[5,4.1]$. To see that $X$ can be given a complex structure, note that there is an open covering $\left\{U_{n}: n \in \mathbf{N}^{*}\right\}$ of $X$ with the subsequent properties: For each $n \in \mathbf{N}^{*}, U_{n}$ is a subset of $X_{n}$, and the complex charts on $X$ defined by the $U_{n}$ can be glued together to form a complex structure $O$ on $X[4, \mathrm{VA} 7]$.

In order to calculate the spectrum of the algebra $A:=\mathcal{O}(X)$, take countably many copies $\mathbf{C}_{n}^{2}, n \in \mathbf{N}$, of $\mathbf{C}^{2}$ such that the first copy $\mathbf{C}_{0}^{2}:=\mathbf{C}^{2} \times\{0\}$ is fixed and the other copies $\mathbf{C}_{n}^{2}:=\left\{\alpha_{\varphi(n)}\right\} \times \mathbf{C}^{2}, n \geq 1$, are transversal to the first copy. Let $Y:=\bigsqcup_{n \in \mathbf{N}} \mathbf{C}_{n}^{2}$ denote their disjoint union with the natural complex structure. $A$ is topologically isomorphic to

$$
\left\{\left(f_{n}\right)_{n \in \mathbf{N}} \in \prod_{n=0}^{\infty} O\left(\mathbf{C}_{n}^{2}\right):\left.f_{n}\right|_{\{z=0\}}=\left.f_{0}\right|_{\left\{x=\alpha_{\varphi(n)}\right\}}, n \geq 1\right\}
$$

and can therefore be viewed as a function algebra on $Y$. 
LEMMA 1. Let $\chi: Y \rightarrow S_{c}(A)$ be the canonical map assigning to every point $y \in Y$ the point evaluation $\hat{y}$ defined by $\hat{y}(f):=f(y)$ for $f \in A$. The spectrum $S_{c}(A)$ is not locally compact at $\hat{y}$ for any $y \in \mathbf{C}_{0}^{2}$.

ProOF. Let $Q$ denote the quotient of $Y$ by the equivalence relation $R_{\chi}$ associated to the map $\chi . Q$ is obtained by identifying the complex line $\left\{x=\alpha_{\varphi(n)}\right\}$ in $\mathbf{C}_{0}^{2}$ with the complex line $\{z=0\}$ in $\mathbf{C}_{n}^{2}$ for every $n \geq 1$.

In $\mathbf{C}_{0}^{2}$, along each line $\left\{x=\alpha_{\varphi(n)}\right\}$ countably many planes are attached, because there are countably many preimages of $\varphi(n)$. Consequently, $\mathbf{C}_{0}^{2}$ is not locally compact along the line $\left\{x=\alpha_{\varphi(n)}\right\}$ for every $n \geq 1$. Since the sequence $\left(\alpha_{n}\right)_{n \in \mathbb{N}}$ is dense in $\mathbf{C}$, the plane $\mathbf{C}_{0}^{2}$ is not locally compact along this line. Such lines are dense in $\mathbf{C}_{\varphi_{1}(n)}^{2}$, implying that $\mathbf{C}_{\varphi_{1}(n)}^{2}$ is not locally compact anywhere. If $p: Y \rightarrow Q$ is the projection, then $Q$ is not locally compact at $p(y)$ for any $y \in \mathbf{C}_{0}^{2}$.

Let $\bar{\chi}: Q \rightarrow S_{c}(A)$ be the unique map making the diagram

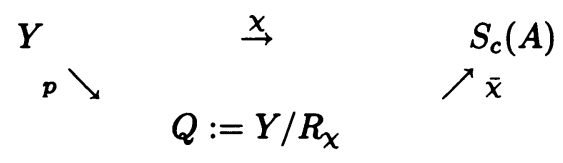

commutative. Since $\bar{\chi}$ is continuous, the assertion of Lemma 1 follows if $\bar{\chi}$ is proper, i.e. inverse images of compact sets are compact.

To prove that the map $\bar{\chi}$ really is proper, the fact that it is surjective will be used. This, in turn, can be seen as follows. $A$ is the strongly dense inverse limit of the Fréchet algebras

$$
A_{m}:=\left\{\left(f_{0}, \ldots, f_{m}\right) \in \prod_{n=0}^{m} O\left(\mathbf{C}_{n}^{2}\right):\left.f_{n}\right|_{\{z=0\}}=\left.f_{0}\right|_{\left\{x=\alpha_{\varphi(n)}\right\}}, 1 \leq n \leq m\right\}
$$

for $m \in \mathbf{N}$ with respect to the surjective maps

$$
\pi_{m}: A_{m+1} \rightarrow A_{m}, \quad\left(f_{0}, \ldots, f_{m+1}\right) \mapsto\left(f_{0}, \ldots, f_{m}\right) .
$$

According to a theorem of Arens [1, 5.21], the surjectivity of the projection $\sigma_{m}: A \rightarrow$ $A_{m}$ onto the first $m+1$ components implies that, in the category of sets, $S_{c}(A)$ is the direct limit of the system $\left(S_{c}\left(A_{m}\right), \pi_{m}^{\prime}\right)$ where $\pi_{m}^{\prime}: S_{c}\left(A_{m}\right) \rightarrow S_{c}\left(A_{m+1}\right)$ is the transposition $\psi \mapsto \psi \circ \pi_{m}$. The spectrum $S_{c}\left(A_{m}\right)$ is homeomorphic to the Stein space $X_{m}^{\prime}$ which is obtained from the disjoint union $\bigsqcup_{n=0}^{m} \mathbf{C}_{n}^{2}$ when the line $\left\{x=\alpha_{\varphi(n)}\right\}$ in $\mathbf{C}_{0}^{2}$ is identified with the line $\{z=0\}$ in $\mathbf{C}_{n}^{2}$ for $1 \leq n \leq m[3,1.5]$. Thus, $\bar{\chi}$ is surjective.

Now it can be shown that $\bar{\chi}$ is proper. Let $K$ be a compact subset of $S_{c}(A)$. There is an $m \in \mathbf{N}$ with

$$
K \subset \sigma_{m}^{\prime}\left(S_{c}\left(A_{m}\right)\right),
$$

where $\sigma_{m}^{\prime}: S_{c}\left(A_{m}\right) \rightarrow S_{c}(A)$ is the transportation. If this were not the case, there would be a sequence $\left(x_{m}\right)_{m \in N}$ in $K$ such that, when considered as a sequence in $Q$ via the bijective map $\bar{\chi}$, the point $x_{m}$ would lie in $\mathbf{C}_{m}^{2}$ but not on the line $\{z=0\}$. Cartan's Theorem A would then ensure the existence of a fucntion $f_{m} \in O\left(\mathbf{C}_{m}^{2}\right)$ with

$$
f_{m}\left(x_{m}\right)=m \text { and } f_{m}=0 \text { on }\{z=0\}
$$


for every $m \geq 1$. Setting $f_{0}:=0$, an element $f:=\left(f_{n}\right)_{n \in \mathbf{N}}$ of $A$ would exist which is unbounded on the sequence $\left(x_{m}\right)_{m \in \mathbf{N}}$. This is a contradiction, since $f$ can be identified with its Gelfand transform which is bounded in $K$. Consequently, an $m \in \mathbf{N}$ exists with the property $(*)$. Since $\sigma_{m}^{\prime}$ is a topological embedding, $K$ can be considered as a compact subset of $X_{m}^{\prime}$ and hence of $Q$, proving that $\bar{\chi}$ is proper. This completes the proof of Lemma 1 .

Second step of the construction. Let $\left(\alpha_{n}\right)_{n \in \mathbf{N}}, D$ and $D_{0}:=D \times\{0\}$ be as in the first step of the construction. Countably many copies $D_{n}, n \geq 1$, of $D$ which are either parallel or transverse to $D_{0}$ will be defined and attached together by means of a surjective map $\varphi: \mathbf{N} \rightarrow \mathbf{N} \times \mathbf{N}, \varphi(0)=(0,0), n \mapsto\left(\varphi_{1}(n), \varphi_{2}(n)\right)$, where each value is assumed countably often and $\varphi_{i}(n)<n$ is true for every $n \in \mathbf{N}^{*}$ and for $i=1,2$. Such a map is given, for example, by composing the map $\mathbf{N}^{*} \rightarrow \mathbf{N}$ used in the first step with a bijective map $\psi: \mathbf{N} \rightarrow \mathbf{N} \times \mathbf{N}, n \mapsto\left(\psi_{1}(n), \psi_{2}(n)\right)$, satisfying $\psi_{i}(n) \leq n$ for $n \in \mathbf{N}$ and $i=1,2$.

For $n \geq 1, D_{n}$ will be defined by induction to be transverse to $D_{\varphi_{1}(n)}$. Let $D_{1}$ be as in the first step of the construction with $\alpha_{0}:=0$. Call that part of $D_{1}$ in the $y z$-plane $D^{\prime}$, i.e. $D_{1}=\{0\} \times D^{\prime}$. If $D_{m}$ has been defined to be parallel or transverse to $D_{0}$ and transverse to $D_{\varphi_{1}(m)}$ for $1 \leq m \leq n-1$, then $D_{\varphi_{1}(n)}$ is either parallel or transverse to $D_{0}$, since $\varphi_{1}(n) \leq n-1$. In the first case define $D_{n}:=\left\{\alpha_{\varphi_{2}(n)}\right\} \times D^{\prime}$ and in the second case set $D_{n}:=D \times\left\{\alpha_{\varphi_{2}(n)}\right\}$. along

The idea now is to obtain a complex space $X$ by identifying each $D_{n}$ with $D_{\varphi_{1}(n)}$

$$
R_{n}:=\left\{(x, y, z) \in \mathbf{C}^{3}: x=\alpha_{\varphi_{2}(n)}, n+\frac{1}{2}<|y|<n+1, z=\alpha_{\varphi_{2}\left(\varphi_{1}(n)\right)}\right\},
$$

if $D_{\varphi_{1}(n)}$ is parallel to $D_{0}$ or along

$$
R_{n}:=\left\{(x, y, z) \in \mathbf{C}^{3}: x=\alpha_{\varphi_{2}\left(\varphi_{1}(n)\right)}, n+\frac{1}{2}<|y|<n+1, z=\alpha_{\varphi_{2}(n)}\right\},
$$

if $D_{\varphi_{1}(n)}$ is transverse to $D_{0}$. Because $\left|\alpha_{\varphi_{2}\left(\varphi_{1}(n)\right)}\right|<n+1$ and $\left|\alpha_{\varphi_{2}(n)}\right|<n+1$ hold, $R_{n}$ is an analytic subset of $D_{n}$ as well as of $D_{\varphi_{1}(n)}$, and such an identification is possible [6].

To be more exact, $X$ will again denote the direct limit of expanding system of complex spaces $X_{n}, n \geq 1$, defined by induction as follows:

$$
X_{1}:=D_{0}+_{R_{1}} D_{1}, \quad X_{n}:=X_{n-1}+_{R_{n}} D_{n} .
$$

As before, $X$ is a complex space $[5,4]$.

The spectrum of $A:=O(X)$ is determined by considering in $\mathbf{C}^{3}$ countably many copies $\mathbf{C}_{n}^{2}, n \in \mathbf{N}$, of $\mathbf{C}^{2}$ parallel to $\mathbf{C}^{2} \times\{0\}$ or to $\{0\} \times \mathbf{C}^{2}$ which are defined by induction. Let $\mathbf{C}_{0}^{2}:=\mathbf{C}^{2} \times\{0\}$ and $\mathbf{C}_{1}^{2}:=\{0\} \times \mathbf{C}^{2}$. If $\mathbf{C}_{m}^{2}$ has already been defined for $1 \leq m \leq n-1$, then $\mathbf{C}_{\varphi_{1}(n)}^{2}$ is either parallel or transverse to $\mathbf{C}_{0}^{2}$. If the former is true, put $\mathbf{C}_{n}^{2}:=\left\{\alpha_{\varphi_{2}(n)}\right\} \times \mathbf{C}^{2}$ and otherwise define $\mathbf{C}_{n}^{2}:=\mathbf{C}^{2} \times\left\{\alpha_{\varphi_{2}(n)}\right\} . A$ is topologically isomorphic to the set of elements $\left(f_{n}\right)_{n \in \mathbb{N}} \in \prod_{n=0}^{\infty} O\left(\mathbf{C}_{n}^{2}\right)$ satisfying the following conditions for all $n \geq 1$ :

$$
\left.f_{n}\right|_{\left\{z=\alpha_{\varphi_{2}\left(\varphi_{1}(n)\right)}\right\}}=\left.f_{\varphi_{1}(n)}\right|_{\left\{x=\alpha_{\varphi_{2}(n)}\right\}}
$$

when $\mathbf{C}_{n}^{2}$ is transverse to $\mathbf{C}_{0}^{2}$ and otherwise

$$
\left.f_{n}\right|_{\left\{x=\alpha_{\varphi_{2}\left(\varphi_{1}(n)\right)}\right\}}=\left.f_{\varphi_{1}(n)}\right|_{\left\{z=\alpha_{\varphi_{2}(n)}\right\}} .
$$


LEMMA 2. The spectrum $S_{c}(A)$ is not locally compact anywhere.

Proof. Denote by $Y:=\coprod_{n \in \mathbf{N}} \mathbf{C}_{n}^{2}$ the disjoint union of the planes $\mathbf{C}_{n}^{2}$ with the natural complex structure; $A$ is a subalgebra of $O(Y)$. Let $\psi: Y \rightarrow S_{c}(A)$ be the map $y \mapsto \hat{y}$ for $\hat{y}(f):=f(y)$. The quotient $Q$ of $Y$ by the equivalence relation $R_{\chi}$ given by $\chi$ is not locally compact at any point. To verify this, notice that $Q$ is obtained from $Y$ by identifying the line

$$
\left\{z=\alpha_{\varphi_{2}\left(\varphi_{1}(n)\right)}\right\} \quad \text { resp. } \quad\left\{x=\alpha_{\varphi_{2}\left(\varphi_{1}(n)\right)}\right\}
$$

in $\mathbf{C}_{n}^{2}$ with the line

$$
\left\{x=\alpha_{\varphi_{2}(n)}\right\} \quad \text { resp. } \quad\left\{z=\alpha_{\varphi_{2}(n)}\right\}
$$

in $\mathbf{C}_{\varphi_{1}(n)}^{2}$ for every $n \geq 1$; the choice of the line depends upon whether $\mathbf{C}_{\varphi_{1}(n)}^{2}$ is parallel or transverse to $\mathbf{C}_{0}^{2}$. Since there are countably many preimages of $\varphi(n)$, there are countably many planes attached to $\mathbf{C}_{\varphi_{1}(n)}^{2}$ along the line given by $x=$ $\alpha_{\varphi_{2}(n)}$ resp. $z=\alpha_{\varphi_{2}(n)}$. Hence, $\mathbf{C}_{\varphi_{1}(n)}^{2}$ is not locally compact along this line. Such lines are dense in $C_{\varphi_{1}(n)}^{2}$, implying the $C_{\varphi_{1}(n)}^{2}$ is not locally compact anywhere. Because $\varphi$ is surjective, no plane $\mathbf{C}_{n}^{2}$ has a point at which it is locally compact. Thus, $Q$ is not locally compact anywhere.

Let $\bar{\chi}: Q \rightarrow S_{c}(A)$ be the canonical map induced by $\chi$. As in Lemma 1 , it follows from a theorem of Arens [1, 5.21] that the continuous injective map $\bar{\chi}$ is surjective. Together with Cartan's Theorem A, this implies that $\bar{\chi}$ is proper, proving Lemma 2.

\section{REFERENCES}

1. R. Arens, Dense inverse limit rings, Michigan Math. J. 5 (1958), 169-182.

2. J. Bingener and U. Storch, Resträume zu analytischen Mengen in Steinschen Räumen, Math. Ann. 210 (1974), 33-53.

3. O. Forster, Primärzerlegung in Steinschen Algebren, Math. Ann. 154 (1964), 307-329.

4. R. Gunning and H. Rossi, Analytic functions of several complex variables, Prentice-Hall, Englewood Cliffs, N.J., 1965.

5. V. Hansen, Some theorems on direct limits of expanding sequences of manifolds, Math. Scand. 29 (1971), 5-36.

6. B. Kaup, Über Kokerne und Pushouts in der Kategorie der komplex-analytischen Räume, Math. Ann. 189 (1970), 60-76.

7. B. Kramm, Analytische Struktur in Spektren-ein Zugang über die $\infty$-dimensionale Holomorphie, J. Funct. Anal. 37 (1980), 249-270.

INSTITUT FÜR MATHEMATIK DER TECHNISCHEN UNIVERSITÄT MÜNCHEN, POSTFACH 202420, D-8000 MÜNCHEN 2, FEDERAL REPUBLIC OF GERMANY

Université de Paris Vi, Mathématiques, 4, Place Jussieu, 75230 Paris Cedex 05, FRANCE 